\title{
Solutions of Strong-Coupling Meson Field Theory with a Hard Core for Elastic Nucleon-Nucleon Interaction
}

\author{
SIEGFRIED WAGNER* \\ II. Institut für Theoretische Physik der Universität, Hamburg, Germany \\ and \\ Deutsches Elektronen-Synchrotron (DESY), Hamburg, Germany
}

(Received 29 July 1968; revised manuscript received 9 September 1968)

\begin{abstract}
In Wentzel's strong-coupling solutions of the fixed-source meson field theory, the nucleon isobars are derived for pion-nucleon interaction. In the limit of strong coupling, the exact solutions of the two-source field equations were also obtained; they contain the transitions between an infinite set of isobaric twonucleon states. Using the matrix representation given by Fierz, these solutions can be expressed as a coupled system of Schrödinger equations containing infinite matrices for the central and tensor potentials as well as an isobar mass-breaking term. In addition, a core potential of the Wigner type was derived for small separations of the sources; this potential is here idealized as a hard core, independent of the state. This theory contains three parameters: the pion coupling constant $g^{2}$, the $N_{33}{ }^{*}$ isobar excitation energy $E_{r}$, and the core radius $r_{c}$. Choosing the values $g^{2}=13, E_{r}=300 \mathrm{MeV}$, and $r_{c} \approx 0.55 \mathrm{~F}$ for these parameters, excellent agreement is obtained for the $S$-wave phase shifts throughout elastic energy region. The high-angular-momentum phase shifts approach one-pion-exchange phase shifts, and the intermediate phase shifts agree qualitatively below $100 \mathrm{MeV}$, except for the triplet $P$. The deviations result essentially from the neglect of the core structure, the absence of spin-orbit forces, and relativistic effects.
\end{abstract}

\section{INTRODUCTION}

$\mathrm{T}$ is known from phenomenology that the elastic $N-N$ interaction can be described by static potentials, and that there are three distinct kinds of them. First, the long-range forces (for distances greater than $2 \mathrm{~F}$ ) are already quantitatively described by the one-pion-exchange contribution (OPEC) potentials; these forces are attractive or repulsive, depending on the quantum number of the state considered. Second, the core is described by forces of the range $\approx 0.7 \mathrm{~F}$ which are very strong and always repulsive. In addition, there are spin-orbit forces derived from these short-range potentials. Contrary to the $\rho$-meson dominance established in other kinds of experiments, the isospin-zero $\omega$-meson potential is here obtained as the strongest one, and therefore the shortrange forces do not depend very much on the state. Third, there are medium-range forces, always attractive, which mainly account for the rather high singleteven phase shifts. Usually they are either partly explained by two-meson exchange terms, ${ }^{1}$ or they are described in the one-boson-exchange (OBE) model by the introduction of hypothetical scalar mesons, ${ }^{2}$ but in the first case it was not possible to obtain convergent series in terms of multiparticle meson states, and in the second case the scalar mesons (which should be the

* Work supported by the Deutsche Forschungsgemeinschaft and Deutsches Elektonen-Synchrotron, DESY (Hamburg).

1 D. Amati, E. Leader, and B. Vitale, Nuovo Cimento 17, 68 (1960); 18, 409 (1960); E. Lomnon and H. Feshbach, Ann. Phys. (N. Y.) 29, 19 (1964); Rev. Mod. Phys. 39, 611 (1967); S. Furuichi and W. Watari, Progr. Theoret. Phys. (Kyoto) 34, 594 (1965); 36, 348 (1966).

${ }_{2}^{2}$ N. Hoshizaki, S. Otsuki, W. Watari, and M. Yonezawa, Progr. Theoret. Phys. (Kyoto) 27, 1199 (1962); R. A. Bryan and B. L. Scott, Phys. Rev. 135, B434, (1964); A. E. S. Green and T. Sawada, Rev. Mod. Phys. 39, 594 (1967); G. Köpp, ibid. 39, 640 (1967); A. Scotti and D. V. Wong, Phys. Rev. 138, B145 (1965). most important particles other than pions to explain nuclear forces) could hardly be found in experiments.

The strong-coupling meson field theory derives all three kinds of forces from a static pion field and from the assumption that the nucleons can be described as extended sources. The long-range OPEC-type interaction is obtained for the phase shifts with high angular momentum, the intermediate-range forces are created via excitation of nucleon isobars, and the Wigner-type core interaction is derived in the intersection region of the two sources. There is no need for scalar mesons in this theory, and possibly the vector mesons need not be introduced explicitly. In our calculation, the longrange and intermediate-range forces are taken into account quantitatively, but the short-range interaction is idealized by a hard core.

After Wentzel ${ }^{3}$ had introduced the strong-coupling concept in 1940, this method was applied to the $N$-N interaction by Pauli, Dancoff, and Serber, ${ }^{4}$ again by Wentzel and his school, ${ }^{5}$ and also by Schwinger and Oppenheimer ${ }^{6}$ in different approaches. They all obtained the same expression for the potentials in the case of the pseudoscalar symmetrical theory. Fierz ${ }^{7}$ developed the matrix representation of the strong-coupling potentials.

The numerical results of this first period did not agree with experiments because of the selection of a value of $50-\mathrm{MeV}$ isobar excitation energy and because

${ }^{3}$ G. Wentzel, Helv. Phys. Acta 13, 169 (1940).

4 W. Pauli and S. M. Dancoff, Phys. Rev. 62, 85 (1942); W. Pauli, Meson Theory of Nuclear Forces (Interscience Publishers, Inc., New York, 1946); W. Pauli and S. Kusaka, Phys. Rev. 63, 400 (1943); R. Serber and S. M. Dancoff, ibid. 63, 143 (1943).

5 G. Wentzel, Helv. Phys. Acta 16, 551 (1943); M. Fierz and

G. Wentzel, ibid. 17, 216 (1944); A. Houriet, ibid. 18, 473 (1945); F. Coester, ibid. 17, 35 (1944); also Refs. 7 and 8.

${ }_{6}^{6}$ J. R. Oppenheimer and J. Schwinger, Phys. Rev. 60, 150 (1941).

${ }^{7}$ M. Fierz, Helv. Phys. Acta 17, 181 (1944) ; 18, 158 (1945). 
of unpermissible approximations. Villars ${ }^{8}$ then discovered that good results could be obtained by assuming a greater value of excitation energy. Finally, Houriet and Héritier ${ }^{9}$ assumed an experimental value of 300 $\mathrm{MeV}$. They included only the isobars with the lowest excitation energy, i.e., the states $\left(N+N_{33}{ }^{*}\right)$ with 300 $\mathrm{MeV}$ for the singlet, and $\left(N_{33}{ }^{*}+N_{33}{ }^{*}\right)$ with $600 \mathrm{MeV}$ for the triplet. A hard-core potential was introduced to account for the short-range forces. Good agreement was obtained for the singlet and triplet effective-range parameters and for the deuteron. This theory was then extended to deal with $N-N$ scattering in the elastic energy region (see Refs. 10-12).

Section 2 contains the strong-coupling solutions of the two-source meson field theory. In Sec. 2 A. the long-range and intermediate-range solutions are given. The short-range forces are discussed in Sec. $2 \mathrm{~B}$. section 3 contains a discussion of the calculated phase shifts in comparison with the experimental phase-shift analysis. In Appendix A, the structure of the intermediate-coupling solutions is discussed, and the contribution of the different isobaric channels is analyzed. Appendix B contains a comparison of the strongcoupling results with the isobar contributions in other theories, including a discussion of isobar potentials.

\section{STRONG-COUPLING SOLUTIONS OF TWO- SOURCE MESON FIELD THEORY}

In strong-coupling theories, the nucleons are described as fixed extended sources; this is why they cannot be formulated in a Lorentz-invariant way. But the meson field is, of course, treated relativistically, and by applying second quantization. In the limit of very strong coupling, as well as in the opposite limit of weak coupling, the exact solutions of the field equations have been derived. The weak-coupling solutions are identical with the familiar point-source solution (OPEC) in the Born approximation, except for a renormalization factor. The complete strong-coupling solutions contain an infinite set of stable isobaric nucleon states. The physical case of rather great but finite coupling lies in between, but tends more towards the limit of strong coupling. This is discussed in more detail in Appendix A.

The Hamiltonian of the fixed extended-source symmetrical pseudoscalar-meson field theory with pseudoscalar coupling is

$$
\mathfrak{H C}=\mathfrak{H C}_{0}+\mathfrak{H C}_{I},
$$

${ }^{8}$ F. Villars, Helv. Phys. Acta 19, 323 (1946).

${ }^{9}$ A. Houriet and C. A. Héritier, Helv. Phys. Acta 35, 414 (1962).

${ }^{10} \mathrm{~A}$. Achour, F. Ade, and S. Wagner, theses at the University of Fribourg, Switzerland, 1967 (unpublished).

${ }^{11} \mathrm{~S}$. Wagner and P. Winiger, Helv. Phys. Acta (to be published).

${ }^{12}$ S. Wagner, DESY Report No. 68/9, 1968 (unpublished). where

$$
\begin{aligned}
\mathfrak{K}_{0}=\frac{1}{2} \sum_{\alpha=1}^{3} \int d V\left(\pi_{\alpha}{ }^{2}-\nabla^{2} \phi_{\alpha}+\mu^{2} \phi_{\alpha}\right), \\
\mathfrak{K}_{I}=-(4 \pi)^{1 / 2} \frac{f}{\mu} \sum_{A=1}^{2} \sum_{\alpha=1}^{3} \sum_{i=1}^{3}{\sigma_{i}}^{(A)} \tau_{\alpha}{ }^{(A)} \\
\times \int d V U\left(r_{i}\right) \nabla_{i}{ }^{(A)} \phi_{\alpha},
\end{aligned}
$$

with $\mu=m_{\pi} c / \hbar . \phi_{\alpha}$ and $\pi_{\alpha}$ are the canonical meson field operators obeying the familiar boson commutation rules. $\sigma_{i}{ }^{(A)}$ and $\tau_{\alpha}{ }^{(A)}$ are the Pauli spin matrices of spin and isospin of the two sources $A=1$ and 2. The coupling constant $f$ is dimensionless. $U\left(r_{i}\right)$ is a source function, ${ }^{13}$ normalized to one.

\section{A. Long-Range and Intermediate-Range Solutions}

Only the results of the strong-coupling calculations are given here. The derivation can be found in Wentzel's ${ }^{5}$ and Fierz's ${ }^{7}$ original papers, and in Pauli, Dancoff, and Serber's ${ }^{4}$ papers on the two source problem. According to Wentzel's approach - which is essentially different from Pauli's and also from the classical field-theory treatment of Schwinger ${ }^{6}$-a set of canonical transformations is applied to the Hamiltonian of Eq. (1) so that the interaction part is diagonalized with respect to spin and isospin. The lowest-energy eigenstates of the transformed Hamiltonian are then found by variational methods. Then in the limit of strong coupling, i.e., with

$$
f^{2} \gg a \mu,
$$

where $a$ is the range of the source function $U\left(r_{i}\right)$, the Hamiltonian reduces to a sum of four terms:

$$
\mathfrak{H C}=\mathfrak{H}_{\text {self }}+\mathcal{H}_{\text {dyn }}+\mathcal{H}_{\text {rot }}+\mathfrak{H}_{\text {pot }} \text {. }
$$

The first term in (3) is the constant self-energy of the field for infinite separations of the two sources.

The second term,

$$
\mathfrak{H}_{\mathrm{dyn}}=-\left(\hbar^{2} / 2 m_{N}\right) \nabla^{2},
$$

is the familiar nonrelativistic dynamical operator.

When the separation of the two sources is large enough, so that there is no overlapping, the third term becomes

$\mathcal{H}_{\text {rot }}=\epsilon \sum_{A=1}^{2}\left[\left(\mathscr{L}^{(A)}\right)^{2}-\left(S^{(A)}\right)^{2}\right], \epsilon=a \mu^{2} / f^{2}$ for $a \mu \ll 1$,

where $\left(\mathscr{L}^{(A)}\right)^{2}$ is the differential operator of the spherical

${ }^{13}$ In the prior strong-coupling paper (Refs. 3-7) it was assumed that the range of the source function is equal to the proton Compton wavelength. Therefore the numerical results disagreed with the experiments. However, it is permissible to assume a greater range on account of the pionic form factor of the nucleon, since in the derivation it is only necessary that the range be smaller than the pion Compton wavelength. 
rotator, and where $\left(S^{(A)}\right)^{2}$ is attributed to the spin of the nucleon source. The eigenvalues are

$$
E_{r}=\epsilon \sum_{A=1}^{2}\left[j_{A}\left(j_{A}+1\right)-\frac{3}{2}\right], \quad j_{A}=\frac{1}{2}, \frac{3}{2}, \frac{5}{2}, \cdots .
$$

In these states the inner part of the meson cloud is bound to the spin of the sources, and these mesons rotate about their respective nucleon centers. The quantum number $j_{A}$ is then the vector sum of the nucleon spin and the angular momentum of the spinning meson cloud, and $1 / E$ can be interpreted in classical field theory as the moment of inertia of a rigid rotator. Hence the nucleon together with the bound-meson cloud appears as one particle with the spin $j_{A}$. In the pseudoscalar symmetrical case the isospin quantum number is found to be equal to the spin value in each state:

$$
t_{A}=j_{A} .
$$

These nucleon isobars can be identified with the experimental ones. The states with $j_{A}=\frac{1}{2}$ and $j_{A}=\frac{3}{2}$ are attributed to the nucleon and the $N_{33}{ }^{*}$ resonance, respectively; the higher spin isobars cannot be attributed to experimentally known nucleon resonances. ${ }^{14}$

The last term in (3) is the distance-dependent part of the static self-energy. In the limit of strong coupling (2), and for sufficiently large separations of the sources the meson states split into three orthogonal parts: the bound meson states of each source and the free meson states. There are no interference terms between them. In this case a simple expression can be derived for the interaction energy:

$$
\begin{aligned}
\mathfrak{H}_{\mathrm{pot}}=V_{12}=\frac{f^{2}}{\mu} \sum_{i=1}^{3} \sum_{j=1}^{3} \sum_{\alpha=1}^{3} S_{i \alpha}{ }^{(1)} S_{j \alpha}{ }^{(2)} & \frac{\partial}{\partial x_{i}^{(1)}} \frac{\partial}{\partial x_{j}^{(2)}} \\
& \times \frac{e^{-\mu|x(1)-x(2)|}}{\left|x^{(1)}-x^{(2)}\right|} .
\end{aligned}
$$

The operators $S_{i \alpha}{ }^{(A)}$ depend only on the Euler angles of a generalized spin-isospin space. They are the generators of the Lie group $S U(2) \times S U(2) .{ }^{15}$ All the

${ }^{14}$ The Hamiltonian equation (1) does not contain the concept of strangeness, but it can easily be extended, as has been done by G. Wentzel, Phys. Rev. 125, 771 (1962), and by the group theorists (see Ref. 15). However, strange-particle effects are unimportant in the $N-N$ interaction case, since they only determine the very-short-range forces which are here included in the source function.

${ }_{15}$ The Lie-group properties of Wentzel's strong-coupling solutions for meson-baryon interaction were studied by C. J Goebel, Phys. Rev. Letters 16, 1130 (1966); C. Dullemond and F. J. M. van der Linden, Ann. Phys. (N. Y.) 41, 372 (1967); and other authors. These results are equivalent - as far as the pseudoscalar symmetrical pion field theory goes- to the Fierz representation here adopted. A mass-breaking term is thus obtained without an ad hoc assumption, which is equivalent to the expression in Eq. $\left(5^{\prime}\right)$; however, this spectrum is different for $S U(3)$ representations of mesons and baryons and only the lowest isobars with spin $\frac{3}{2}$ seem to be physically significant. Isobars with negative parity and those with spin quantum number unequal to the isospin do not appear in the strong-coupling limit. half-integer values of spin and of isospin appearing in (7) are those given by Eqs. (5') and (6).

In the fundamental representation the generators $S_{i \alpha}{ }^{(A)}$ are simple products of Pauli matrices, and the weak-coupling solution of the extended-source Hamiltonian (1) takes the form of the perturbation-theoretical OPE potential

$$
\begin{aligned}
& S_{i \alpha}{ }^{(A)} \rightarrow \frac{1}{3} \sigma_{i}{ }^{(A)} \tau_{\alpha}{ }^{(A)} \quad \text { and } \quad V_{12} \rightarrow \frac{1}{9} V_{\mathrm{OPEC}} \\
& \text { for } j_{A}=t_{A}=\frac{1}{2} \text {, }
\end{aligned}
$$

except for the factor $\frac{1}{9}$. This would be the case here if the excitation energy of the isobars were very large compared with the $N-N$ interaction energy. Since this is not so, the isobars must be taken into account, applying Eqs. (7), (5), and $\left(5^{\prime}\right)$ instead of $\left(7^{\prime}\right)$. For the phase shifts with high angular momentum these solutions are obtained approximately, since the isobar effects have a shorter range and are therefore screened by the angular-momentum barrier. Hence the strongcoupling and weak-coupling solutions are identical for the long-range forces.

Fierz ${ }^{7}$ introduced the irreducible basis

$$
n=\left|L, S, j_{1}, j_{2}\right\rangle,
$$

where $L$ is the angular momentum, $S$ is the spin quantum number of the $N-N$ system, and $j_{1}=t_{1}$, $j_{2}=t_{2}$ are the spin quantum numbers of the isobaric nucleon states. The Hamiltonian commutes with total momentum $J$, total isospin $T$, and parity $P$. Therefore the following system of Schrödinger equations results:

$$
\begin{gathered}
\begin{array}{c}
\sum_{n^{\prime}}\left[\left(-\frac{d^{2}}{d x^{2}}+\frac{L_{n}\left(L_{n}+1\right)}{x^{2}}\right) \delta_{n n^{\prime}}+V_{n n^{\prime}} J T P(x)\right] F_{n^{\prime}}{ }^{J T P}(x) \\
={ }^{2} F_{n}{ }^{J T P}(x),
\end{array} \\
\text { with } \begin{array}{c}
x=\mu r, \quad r=\left|\mathbf{r}^{(1)}-\mathbf{r}^{(2)}\right|, \quad \mu=m_{\pi} c / \hbar, \\
\eta=\left(m_{N}{ }^{2} / m_{\pi}\right)\left(\frac{1}{2} E_{d}-E_{r}\right),
\end{array}
\end{gathered}
$$

where $E_{d}$ is the scattering energy in the lab system, and where the isobar excitation energy $E_{r}$ is given by

$$
E_{r}=\epsilon\left[j_{1}\left(j_{1}+1\right)+j_{2}\left(j_{2}+1\right)-\frac{3}{2}\right],
$$

with $\epsilon$ defined as in Eq. (5).

The potential matrices in Eq. (9) are

$$
\begin{gathered}
V_{n n^{\prime}}{ }^{J T P}(x)=\left(m_{N} / m_{\pi}\right) f_{r}{ }^{2}\left\langle j_{1} j_{2}\left|\boldsymbol{\Omega}^{\prime}\right| j_{1}{ }^{\prime} j_{2}{ }^{\prime}\right\rangle \delta_{S S^{\prime}} \delta_{L L^{\prime}}\left(e^{-x} / x\right) \\
+\left(m_{N} / m_{\pi}\right) f_{r}{ }^{2}\left\langle S L j_{1} j_{2}\left|\Theta^{\prime}\right| S^{\prime} L^{\prime} j_{1}{ }^{\prime} j_{2}{ }^{\prime}\right\rangle \\
\times\left(1+3 / x+3 / x^{2}\right) e^{-x} / x . \quad\left(9^{\prime \prime}\right)
\end{gathered}
$$

While the isobar energy $\epsilon$ in Eq. (5) is determined by the full coupling constant $f$, the potentials contain a reduced coupling constant

$$
f_{r}{ }^{2}=\frac{1}{9} f^{2},
$$

because the major part of the meson cloud is bound to the spin of the nucleons. 
The radial dependence in Eq. ( $\left.9^{\prime \prime}\right)$ is the same as that of the OPE potential, but the coefficients of the central and tensor potentials,

$$
\begin{aligned}
& \boldsymbol{\Omega}^{\prime}=3 \boldsymbol{\Omega}\left(T, S, j_{1}, j_{1}{ }^{\prime}, j_{2}, j_{2}{ }^{\prime}\right), \\
& \boldsymbol{\Theta}^{\prime}=9 \mathbf{T}\left(J, T, S, S^{\prime}, L, L^{\prime}, j_{1}, j_{1}{ }^{\prime}, j_{2}, j_{2}{ }^{\prime}\right)-3 \boldsymbol{\Omega},
\end{aligned}
$$

are here infinite matrices containing the transitions to the isobaric two-nucleon states. The matrix elements of $\boldsymbol{\Omega}$ and $\mathrm{T}$ can be computed using the formulas given by Fierz. ${ }^{7}$

The following limitations for the quantum numbers which appear in Eq. (9) were obtained:

$$
\begin{gathered}
\Delta L=0, \pm 2, \quad \Delta S=0, \pm 2, \\
\Delta j_{1}=0, \pm 1, \quad \Delta j_{2}=0, \pm 1, \\
\left|j_{1}-j_{2}\right| \leq S, \quad T \leq j_{1}+j_{2}, \\
|L-S| \leq J \leq L+S, \\
L+S+T \text { is odd. }
\end{gathered}
$$

In the case of elastic $N-N$ scattering only the systems $(J, T, P)$ containing at least one ground-state basis vector with $j_{1}=j_{2}=\frac{1}{2}$ are involved, and the total isospin can only have the values $T=0$ and $T=1$. Symmetrical wave functions can be used. Then, in accordance with the restrictions of Eq. (10), the quantum number

$$
j=j_{1}+j_{2}, \quad j=1,2,3, \cdots
$$

determines unambiguously an isobaric channel with a discrete value of the excitation energy given by Eq. (9').

The value of $\epsilon$ is fixed by identifying the lowest isobaric level $j_{1}=\frac{1}{2}$ and $j_{2}=\frac{3}{2}$ with the state $\left(N+N_{33}{ }^{*}\right)$ of $300-\mathrm{MeV}$ excitation energy ${ }^{16}$ taken from experiment:

$$
\epsilon=100 \mathrm{MeV} \text {. }
$$

Below the threshold of creation, i.e., $E_{d} \geq 600 \mathrm{MeV}$, the system of coupled differential equations (9) decomposes into two types: first, the usual equations of the oscillatory type for the components with both nucleons in the ground state; second, the infinite system of isobaric equations, which are of the exponential type with the boundary condition that the solutions should be zero at infinity.

\section{B. Core Potential}

In the strong-coupling approach, the short-range forces can be represented, as usual, by vector-meson-

\footnotetext{
${ }^{16}$ The lowest channels and their excitation energies are the following:

$E_{r}=0 \quad$ for $j=1$, i.e., the two nucleons in the ground state.

$E_{r}=300 \mathrm{MeV}$ for $j=2$, i.e., the symmetrical state with one nucleon in the ground state, and the other in the $N_{33}{ }^{*}$ state.

$E_{r}=600 \mathrm{MeV}$ for $j=3$, i.e., the two nucleons in the $N_{33}{ }^{*}$ state.

$E_{r}=1100 \mathrm{MeV}$ for $j=4$, i.e., one nucleon in the $N_{33}{ }^{*}$ state, the

$E_{r}=1600 \mathrm{MeV}$ for $j=5$, etc
}

exchange terms. ${ }^{17} \mathrm{It}$ is well known from the one-bosonexchange-contribution (OBEC) model $^{2}$ calculations that the $\omega$ meson and not the $\rho$ meson is dominant, i.e., that the forces are nearly isospin-independent and are repulsive. Forces of this kind are here obtained in the intersection region of the two source functions from the Hamiltonian equation (1) using a pure pion field. Serber and Dancoff ${ }^{4}$ and later Chun $^{18}$ and Houriet ${ }^{19}$ derived a potential for the case in which the two source functions are close together. (In this region-where the source functions overlap - the solutions of Wentzel and Fierz given in Sec. $2 \mathrm{~A}$ are not valid.) The potential depends only on the shape of the source function and is independent of the outer meson field; therefore it is spin-isospin-independent and of the Wigner type. Since spherical symmetric source functions are assumed (this condition may be relaxed if necessary) one gets a central potential. This is here idealized as a hard core

$$
\begin{array}{rlll}
V_{\text {core }}=\infty & \text { for } & r \leq r_{c} \\
& =0 & \text { for } & r>r_{c},
\end{array}
$$

with the core radius $r_{c}$ taken to be state-independent. In both cases, Eqs. (5) and (13), only the range is included, and not the actual form of the source functions. The values $E_{r}$ and $r_{c}$ determined by the range $a$ and the unrenormalized coupling constant $f$ are here treated as independent parameters.

\section{COMPARISON OF CALCULATED PHASE SHIFTS WITH EXPERIMENT}

The coupled system of differential equations Eqs. (9) $-\left(9^{\prime \prime \prime \prime}\right)$ can be solved exactly provided that the number of components is finite. The wave functions are calculated using the iterative procedures developed in Refs. 10 and 11. The only wave functions whose amplitudes do not asymptotically approach zero are those for the channel with no excited nucleons. Thus these wave functions are used for determining the phase shifts by comparing them with the corresponding Bessel functions. In the case of triplet $J \neq L$, the so-called Blatt-Biedenharn eigenphase shifts are determined..$^{20}$

The experimental values are taken from the energyindependent solution of the 1968 Livermore phase-shift analysis. ${ }^{21}$ The Stapp eigenphase shifts were transformed into Blatt-Biedenharn eigenphase shifts. For $330 \mathrm{MeV}$ at isospin $T=0$ the experimental values are

17 Most of Wentzel's strong-coupling work (Ref. 5) deals with vector mesons. Both he and Pauli obtained the strong-coupling solutions of the special M $\phi$ ller-Rosenfeld-Schwinger mixture of a pseudoscalar and vector field. Just recently Wentzel, University of Chicago Report No. EFI-68-24 (unpublished), discussed the general case of $S U(3)$ mixture of pseudoscalar and vector mesons in baryon-meson interaction.

${ }_{18}$ K. W. Chun, Phys. Rev. 112, 973 (1961).

$19 \mathrm{~A}$. Houriet (unpublished).

${ }^{20}$ F. Rohrlich and J. Eisenstein, Phys. Rev. 75, 705 (1949); J. M. Blatt and L. C. Biedenharn, ibid. 86, 399 (1952).

${ }_{21}$ M. H. MacGregor, R. A. Arndt, and M. Wright, Phys. Rev. 169, 1128 (1968); 173, 127 (1968). 


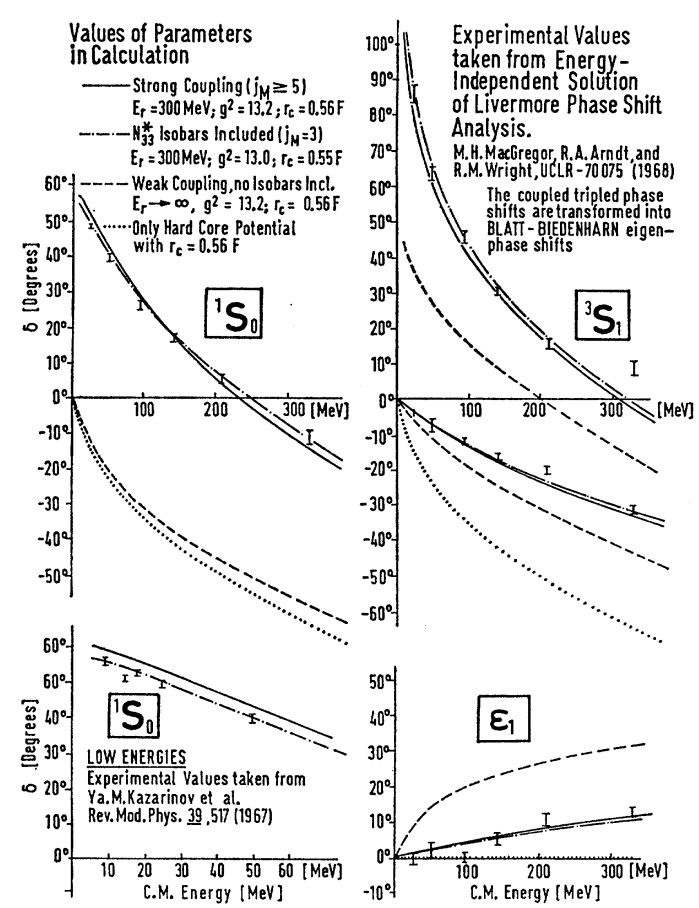

Fig, 1, $S$-wave phase shifts in degrees versus energy in $\mathrm{MeV}$. Blatt-Biedenharn convention for eigenphase shifts The Stapp solutions of the phase-shift analysis were transformed into BlattBiedenharn eigenphase shifts using the formulas of $\mathrm{H}$. Stapp et al., Phys. Rev. 105, 302 (1957)].

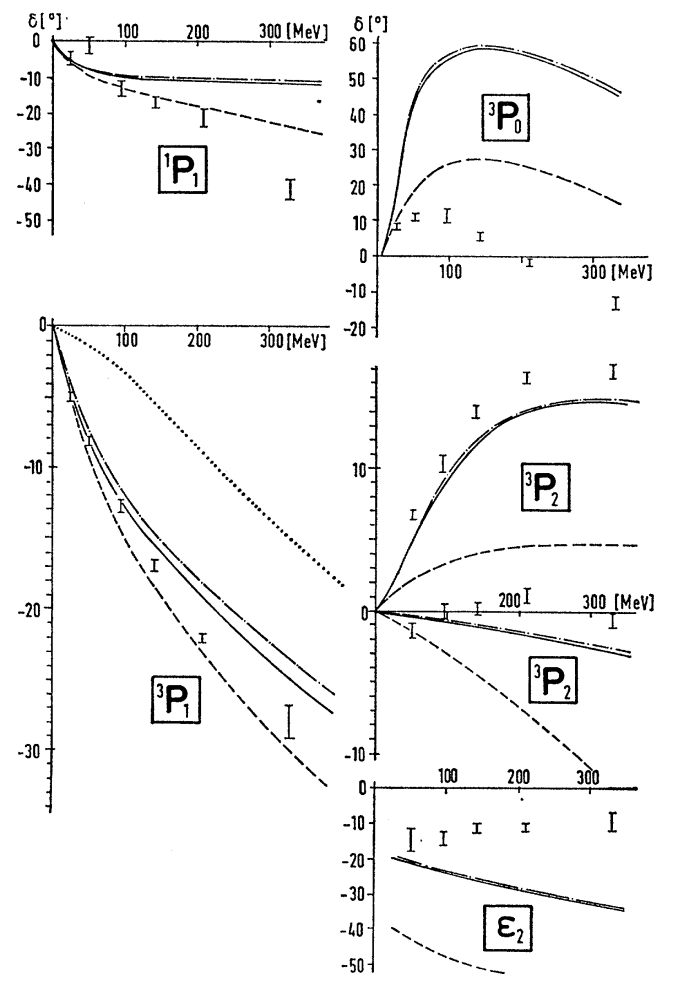

Fig. 2. $P$-wave phase shifts in degrees versus energy in $\mathrm{MeV}$. Blatt-Biedenharn convention for eigenphase shifts.

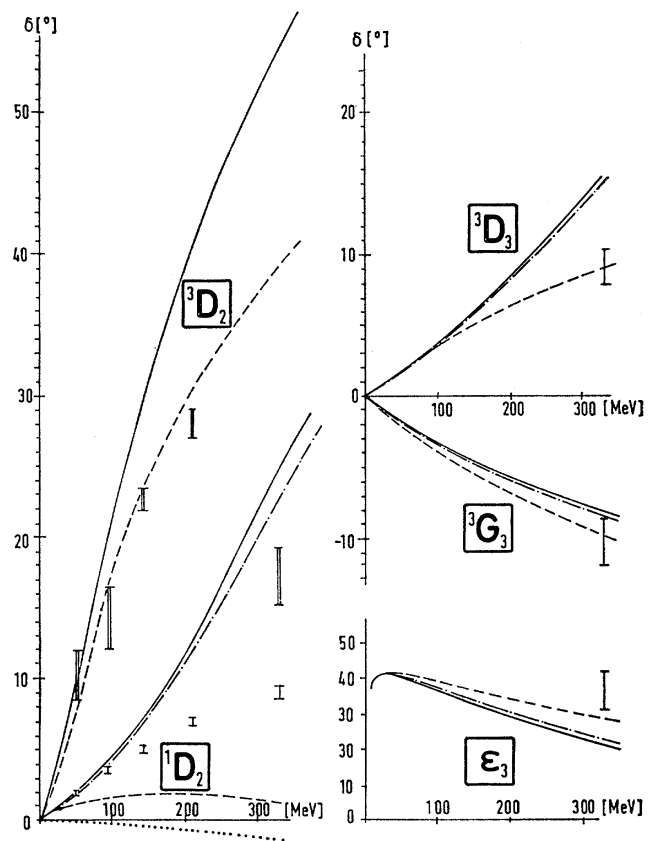

Fig. 3. $D$-wave phase shifts in degrees versus energy in $\mathrm{MeV}$. Blatt-Biedenharn convention for eigenphase shifts.
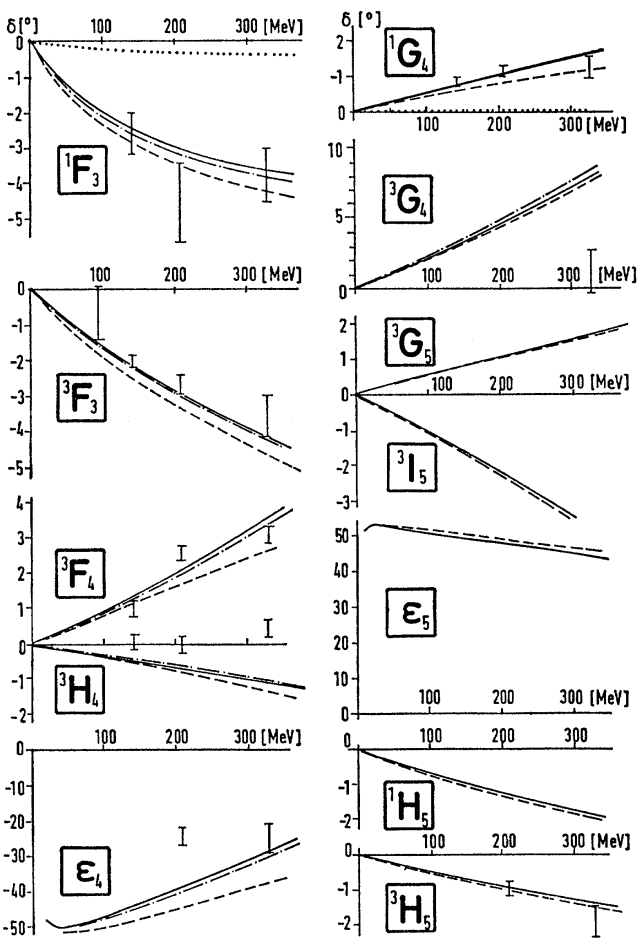

FIG. 4. Phase shifts for high angular momentum in degrees versus energy in MeV. Blatt-Biedenharn convention for eigenphase shifts. 
still in doubt; the disagreement in this case will therefore not be discussed in what follows. Experimental and calculated phase shifts are shown in Figs. 1-4.

The isobar excitation energies were already fixed by assuming the experimental value for the isobaric level to be equal to the mean $N_{33}{ }^{*}$ resonance energy. The other two parameters are determined by the fitting of the ${ }^{1} S_{0}$ phase shift. The shape of this phase shift is mainly due to the core potential (see Fig. 1), and the other potentials cause a parallel shift.

It will be shown in Appendix A that the solutions containing the isobaric states with $j_{1}+j_{2} \leq \frac{5}{2}$ are already practically identical with the limiting strongcoupling solutions containing all isobars. Furthermore it will be assumed that for physical values of the coupling constant even the $\frac{5}{2}$ isobars should be neglected because of their large resonance broadening. Both cases (with and without the inclusion of the spin- $-\frac{5}{2}$ states) are considered here. The parameters obtained are the following:

for $j_{M}=5$ or $j_{M}=7: g^{2}=13.2$ and $r_{c}=0.56 \mathrm{~F},{ }^{22}$ for $j_{M}=3$ : $\quad g^{2}=13.0$ and $r_{c}=0.55 \mathrm{~F}$.

In addition, the weak-coupling phase shifts (with no isobars involved) were calculated for the parameters of the first set, and the phase shift created by the hard-core potential alone is plotted in Figs. 1-4 for comparison.

Figure 1 shows the $S$-wave phase shifts ${ }^{1} S_{0},{ }^{3} S_{1-}{ }^{3} D_{1}$, and $\epsilon_{1}$. With the chosen values for the parameters, the ${ }^{1} S_{0}$ phase shift is in agreement with experiments in the whole energy region. (It was not possible to calculate the wave functions for energies below $5 \mathrm{MeV}$; in this region, the effective-range formalism should be applied as was done in Ref. 9 for the inclusion of lowest isobaric states.) In the strong-coupling case the ${ }^{3} S_{1}$ phase shift is slightly high. However, with the exclusion of the $\frac{5}{2}$ isobars there is complete agreement with experiments for all $S$-wave phase shifts, except for the $330-\mathrm{MeV}$ values of the triplet, which are uncertain in experiments.

The phase shifts with high angular momentum in Fig. 4 are in agreement with experiments for the same parameters as determined for the $S$ waves. Since in this case the angular-momentum barrier screens all shortrange effects including those of the isobars, the OPEC phase shifts are obtained according to Eq. $\left(7^{\prime}\right)$. This justifies the OPEC assumptions in the modified phaseshift analysis.

Deviations occur for the $P$ and $D$ waves, especially for the phase shift ${ }^{3} P_{0}$. Contrary to the phase shifts discussed so far, these intermediate phase shifts depend

\footnotetext{
${ }^{22}$ For comparison, the coupling parameter $g^{2}=\left(2 m_{N} / m_{\pi}\right)^{2} f_{r}{ }^{2}$ is introduced here. The value obtained is somewhat smaller than the one known from $\pi-N$ interaction, which is also used in the recent OBEC fits. The greater $g^{2}=15$, connected with a greater core radius, would cause the slope of the ${ }^{1} S_{0}$ phase shift to become too steep. The pion mass was taken to be that of the charged meson, $m_{\pi}=139.6 \mathrm{MeV}$, thereby enhancing the disagreement in addition to the hard-core idealization.
}

essentially on the structure of the core potential and on the spin-orbit forces, which are neglected in our theory. Qualitatively these effects will move the phase shifts towards the experimental ones. However, the numerical methods implying integral equations with iterative procedures fail to converge when steep core potentials are involved.

\section{ACKNOWLEDGMENTS}

I would like to thank Professor R. Haag for his kind hospitality. This paper is based on work carried out with Professor A. Houriet. For advice on numerical methods applied I would also like to thank Dr. A. Achour, Dr. F. Ade, and P. Winiger from Fribourg, Switzerland. The assistance of the DESY Rechenzentrum is greatly appreciated.

\section{APPENDIX A : INTERMEDIATE-COUPLING SOLUTIONS IN STRONG-COUPLING APPROACH}

Wentzel's strong-coupling condition, Eq. (2), does not mean that solutions of Eq. (1) are only available for the unphysical limit of infinite coupling, but rather that it is the condition for the convergence of an expansion in powers of $1 / f^{2}$. Since the physical coupling constant is large, the first term of this expansion (which is accounted for in our calculations) gives already the main contribution. The higher terms should not essentially change the algebraic properties of these solutions. However, the isobars are then obtained as unstable states, because the bound and unbound meson field operators are no longer uncoupled. Intermediatecoupling solutions of this kind were only obtained for the charged scalar theory and for the $\pi-N$ interaction..$^{23}$

Pauli $^{4}$ derived the criterion according to which only the isobars with low spin (and isospin),

$$
j_{A}<f^{2} / a \mu,
$$

are stable enough to be significant, whereas the higherspin isobars are supposed to hardly contribute. In the case of weak coupling no isobars come into account (as in perturbation theory with point sources), and for infinite coupling all isobars will contribute; for the physical coupling constant only the $\frac{3}{2}$ isobars should be stable enough to be included.

Figure 1 shows indeed that the $S$-wave phase shifts calculated with the inclusion of the $\frac{3}{2}$ isobars alone are improved compared to the phase shift calculated by the inclusion of higher-spin isobars in addition.

Figures 5(a)-5(c) show the phase shifts plotted versus the number $j_{M}$ of included isobaric states. $j_{M}=1$ (no isobars) and $j_{M}=\infty$ (all isobars) are the limiting cases of weak and strong coupling. The main contribution to

${ }^{23}$ This was already the concept of Wentzel's first papers (Refs. 1 and 5) and was later worked out by H. Jahn [Phys. Rev. 126, 824 (1962); Nucl. Phys. 26, 353 (1961)], who used the correct canonical commutation rules. 


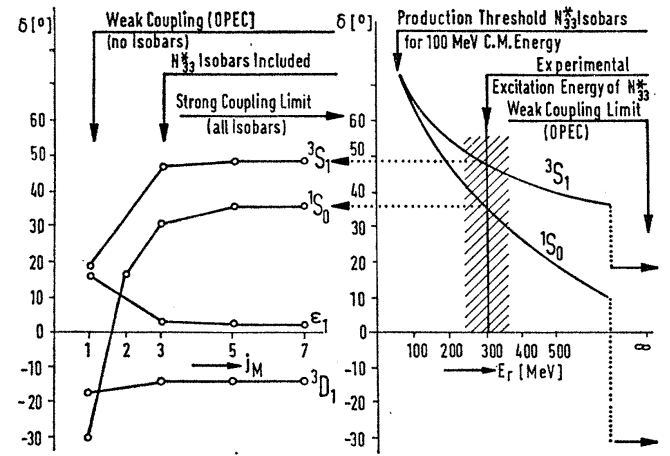

(a)

(d)

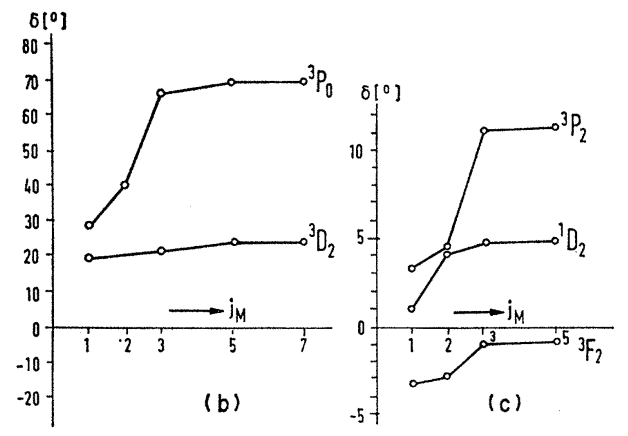

Frg. 5. (a)-(c) Calculated phase shifts at $100-\mathrm{MeV}$ lab energy versus number of isobaric channels included $\left(j_{1}+j_{2} \leq j_{M}\right)$ Parameters of set $N: E_{r}=300 \mathrm{MeV}, g^{2}=14, r_{c}=0.56 \mathrm{~F}$. (d) Calculated phase shifts for $S$ waves at $100-\mathrm{MeV}$ lab energy versus assumed value of $N_{33}{ }^{*}$ excitation energy. Parameters of set $N: j_{M}=5$.

the phase shifts is created in the channel with no excited nucleon, and in the channels with one or both nucleons in the $N_{33}{ }^{*}$ state. Only the $\frac{5}{2}$ isobars still contribute remarkably (which is of importance for the $S$ waves). The higher-spin isobars have a negligible influence, because they do not directly couple to the states with $j \leq 3$ according to the selection rules, Eq. (10).

Figure 5(d) shows the $S$-wave phase shifts for strong coupling $\left(j_{M}=5\right)$ versus the assumed value of the $N_{33}{ }^{*}$ excitation energy $E_{r}$. For $E_{r}=50 \mathrm{MeV}$ (which was the value assumed by Pauli et al. ${ }^{4}$ and by Wentzel et $a l .5$ ), the two $S$-wave phase shifts become identical. The weak-coupling limit-where the isobars have practically no influence-is reached only for $E_{r}>10^{4}$ $\mathrm{MeV}$. Only for the experimental value $E_{r}=300 \mathrm{MeV}$ is the correct splitting of the $S$ waves ${ }^{1} S_{0}$ and ${ }^{3} S_{1}$ obtained. Since this is hardly dependent on the choice of the two other parameters, the physical evidence of the isobars in the $N-N$ interaction seems to be established.

\section{APPENDIX B: $N_{33} *$ CONTRIBUTIONS TO $N-N$ INTERACTION IN OTHER KINDS OF THEORIES AND EFFECTIVE ISOBAR POTENTIALS}

In the OBE models ${ }^{2}$ the intermediate-range interaction is described by the exchange of one or two scalar mesons ; as in Lommon and Feshbach' ${ }^{1}$ boundarycondition model no $N_{33}{ }^{*}$ effects are included. Attempts have also been made to account for a part of the intermediate-range interaction by correlated and uncorrelated two- and three-meson exchange terms, ${ }^{1}$ but they are sufficient only to explain the $L \geq 2$ phase shifts. Scotti and Wong in one of their models as well as Amati, Leader, and Vitale ${ }^{1}$ included parts of the two-pion exchange contributions via $N_{33}{ }^{*}$ poles in the crossed $t$ channel.

In a nonrelativistic $S$-matrix approach, Sugawara and von Hippel ${ }^{24}$ included the isobar levels $\left(N+N_{33}{ }^{*}\right)$ and $\left(N_{33}{ }^{*}+N_{33}{ }^{*}\right)$ as intermediate states in the $s$ channel. They used the experimental $N_{33}{ }^{*}$ excitation energy, and they took the $N_{33}{ }^{*}$ coupling constant from the experimental resonance broadening. Otherwise the effect of resonance broadening was neglected. They obtained a coupled-channel Schrödinger equation. Although the latter calculation seems to have been done without any knowledge of strong-coupling field theory, it is in principle the same approach as ours, apart from some additional approximations made there, such as the neglect of all higher-spin isobars and of all matrix elements which do not couple to the groundstate channel $(N+N)$. Then the infinite-component Schrödinger equation, Eq. (9), reduces to a onecomponent integro-differential equation. However, these simplifications are not allowed for the low- $L$ states such as, e.g., ${ }^{1} S_{0},{ }^{3} P_{0}$, and ${ }^{3} P_{2}$. The main difference in the two approaches is that the isobar spectrum can be calculated in the strong-coupling theory, while it has to be assumed $a d$ hoc in $S$-matrix theory.

Sugawara and von Hippel ${ }^{24}$ derived effective isobar potentials from the coupled-channel Schrödinger equation, which are attractive for all states and are energydependent (only very weakly since no relativistic corrective terms are added). These potentials account for the main part of the intermediate-range interaction, and they can be compared with the scalar meson terms introduced in Ref. 2 ad hoc. The central-potential part is equivalent to a $\sigma$-meson term, and the tensor potential part of the isobar potential may cause something like the $s$-meson contribution.

${ }^{24} \mathrm{H}$. Sugawara and F. von Hippel, Phys. Rev. 172, 1764 (1968). 